\title{
Digital Survey Assessment of Factors Associated with Musculoskeletal Complaints Among US Ophthalmologists
}

\author{
Nicholas E Tan $\mathbb{D}^{\prime}$ \\ Brayden $T$ Wortz ${ }^{2}$ \\ Eric D Rosenberg (iD ${ }^{3}$ \\ Nathan M Radcliffe (iD) 4,5 \\ Preeya K Gupta iD ${ }^{6}$ \\ 'College of Medicine, State University of \\ New York Downstate Health Sciences \\ University, Brooklyn, NY, USA; \\ ${ }^{2}$ Undergraduate College, Wake Forest \\ University, Winston-Salem, NC, USA; \\ ${ }^{3}$ Department of Ophthalmology, \\ New York Medical College, New York, \\ NY, USA; ${ }^{4}$ Department of \\ Ophthalmology, Mount Sinai School of \\ Medicine, New York, NY, USA; \\ ${ }^{5}$ New York Eye Surgery Center, Bronx, \\ NY, USA; ${ }^{6}$ Triangle Eye Consultants, \\ Cary, NC, USA
}

Correspondence: Preeya K Gupta Triangle Eye Consultants, Cary, NC, USA Tel +1919391-7224

Email preeyakgupta@gmail.com
Purpose: To quantify features of musculoskeletal (MSK) complaints among US ophthalmologists and analyze factors related to symptom severity.

Methods: Cornea, glaucoma, retina, and comprehensive practitioners were invited to complete a web-based survey via email. Data on demographics, practice and procedural volumes, wellness activities, job stress, MSK health, the Total Disability Index (TDI), and ergonomics were collected. Significance testing was performed to evaluate factors related to TDI score. Results: Of the 245 ophthalmologists contacted, $58.8 \%(n=144)$ responded at least in part to the survey. Pain episodes were reported in $81.4 \%(n=83)$ of 102 respondents, with $48.1 \%(n=$ $49)$ experiencing daily or weekly attacks. The neck, low back, and shoulders were commonly affected, yet only $57.1 \%(n=20)$ of those with shoulder pain reported a corresponding diagnosis. The mean TDI score was $6.2 \pm 7.3 \%$, indicating minimal disability related to the spine. Demographics, volume metrics, and wellness hours were each not significantly associated with TDI score $(\mathrm{p}>0.05)$. Higher job stress ratings were reported by those who experienced more frequent pain attacks $(p=0.02)$ and those with higher TDI scores $(p=0.001)$. Greater difficulty with clinic, laser, and surgery job tasks was observed in respondents with higher TDI scores $(\mathrm{p}<0.001, \mathrm{p}=0.005$, and $\mathrm{p}<0.001)$ and more bodily pain locations $(\mathrm{p}=0.002, \mathrm{p}=0.002$, and $\mathrm{p}=0.001)$. Respondents who pursued practice modifications $(\mathrm{p}=0.03)$ and treatments $(\mathrm{p}=$ $0.01)$ to reduce or prevent pain had higher mean TDI scores, and $94.2 \%$ of respondents $(n=97)$ were interested in learning more about ergonomics.

Conclusion: Mild MSK complaints were highly prevalent among the surveyed ophthalmologists, and a similar trend could be expected for other US ophthalmologists. Greater frequency and severity of pain may contribute to physician burnout. There may be underdiagnosis of MSK pathology yet also high demand for ergonomic strategies and MSK treatments, suggesting a need for practical solutions.

Keywords: ergonomics, musculoskeletal diseases, occupational diseases, ophthalmologists, surveys and questionnaires

\section{Introduction}

There is increasing interest in identifying and reducing the occupational hazards of practicing ophthalmology. In prior studies, over half of eye specialists surveyed reported musculoskeletal (MSK) complaints, particularly in the neck, back, and upper extremity. ${ }^{1-10}$ Issues have been severe enough to cause some ophthalmologists to decrease their case load or cease conducting surgical procedures. $^{9,10}$ Pain has been reported in both the clinic and surgical settings, with many eye physicians believing that the use of tools like slit lamps, indirect 
ophthalmoscopes, surgical microscopes, and loupes contribute to their symptoms. ${ }^{5,6,10}$ Microsurgery in general appears to affect MSK dysfunction, with suspected mechanisms including sustained forward head posture, muscular fatigue, and awkward positioning caused by equipment. ${ }^{11,12}$ One comparative analysis found that ophthalmologists were not only more likely to experience neck, hand/wrist, or back pain compared to family practitioners, but also more frequently reported highstrain work. $^{8}$

This current study was designed to comprehensively quantify the practice settings, bodily locations, frequency, intensity, and effects of MSK issues among US ophthalmologists, and to explore factors associated with symptom severity. To more precisely define symptom severity and functional impairment than previous research, we utilized the 14 -item Total Disability Index (TDI). ${ }^{13}$ The TDI provides a statistically validated composite measure of pain and disability related to the neck and back. ${ }^{14}$ It was hypothesized that ophthalmologists with larger workloads would have higher TDI scores, and that those with higher TDI scores would experience greater self-reported job stress.

\section{Methods}

IRB exemption for this cross-sectional digital survey study was obtained from Sterling IRB (Atlanta, GA). Participants were informed of the purpose, data usage, anonymity, and voluntariness of the study preceding survey administration for implied consent. No incentives were provided for completion, and once in the survey, a participant could leave at any time. All data was collected anonymously. Conduct of the study was performed in accordance with the standards of the 1964 Helsinki Declaration and its later amendments. The questionnaire was designed on SurveyMonkey and emailed to US ophthalmologists practicing in comprehensive ophthalmology, cornea, glaucoma, and retina. Invitations were sent from August 2020 to January 2021. Email addresses were obtained through academic departments, ophthalmology professional groups, and the personal networks of the authors. A goal of at least 100 respondents was set for this convenience sample, and no power calculations for sample size were made in advance.

The survey included questions relating to demographics, practice volumes, procedural volumes, social history, MSK health, job task difficulties, job stress, the TDI, and ergonomics. MSK complaints were defined as pain, discomfort, or disability episodes. Difficulties with clinic exam, in-office lasers, and surgery due to musculoskeletal symptoms were each self-rated with levels including "does not affect," "makes it slightly more difficult," "moderately more difficult," "significantly more difficult," and "caused me to stop this activity." Job stress was selfrated on a 1-7 scale with 1 indicating "not stressful at all," 4 being "moderately stressful," and 7 equating to "extremely stressful." The TDI was scored out of $100 \%$, with higher percentages indicating greater disability. Scores from $0-20 \%$ indicate mild disability, $21-40 \%$ moderate disability, 41-60\% severe disability, 61-80\% crippling disability, and $81-100 \%$ bed-bound status. The full survey is available in PDF form in Supplemental Material.

Questions were asked relative to the February 2019February 2020 period, in order to avoid confounding effects from the practice changes incurred by the COVID-19 pandemic. To minimize survey dropout, SurveyMonkey's "question piping" feature was used. Piping allowed respondents to received certain questions only when relevant. For instance, if a surgeon selected "retina" as their sole subspecialty, they would not be asked cornea questions later in the survey. For questions skipped due to piping, data was coded as "none," "no," or "0" where appropriate. Due to piping, respondents would receive a range of 25-60 total questions.

The data were analyzed using GraphPad Prism for Mac (v. 8, GraphPad Software) and Statistical Package for the Social Sciences for Mac (v. 27, IBM). Descriptive statistics were compiled. Student's $t$-test, one-way ANOVA, and univariate linear regressions without adjustment were performed to evaluate how demographic factors, practice volumes, surgical volumes, and wellness activities correlated with TDI scores. Univariate ordinal regressions without adjustment tested how TDI scores, number of bodily pain locations, and number of previous MSK diagnoses associated with ophthalmologist job task difficulty. Univariate ordinal regression without adjustment evaluated the relationship between TDI score and job stress, while Student's $t$-test and one-way ANOVA tested how the presence of MSK pain and pain episode frequency, respectively, associated with stress. For the outcomes of workplace changes and treatment use, chi-square testing assessed the impact of MSK pain presence and pain episode frequency. Student's $t$-test determined how TDI scores compared between those who made adjustments for pain and those who had not. Chi-square testing evaluated outcomes related to ergonomic learning, 
interest, and assistance. Continuous values were reported as mean \pm standard deviation. Significance was set at $\mathrm{p}<0.05$.

\section{Results}

In total, 245 ophthalmologists were contacted, with $58.8 \%$ $(n=144)$ answering at least part of the survey. All eligible questions, as determined by question piping, were completed by $40.4 \% \quad(n=99)$ of respondents. Demographic features are compiled in Table 1 . The average respondent was middle-aged, with a healthy Body Mass Index and over 20 years of practice experience. Both age (50.0 \pm $10.4 ; \mathrm{p}=0.21)$ and years in practice $(20.8 \pm 10.4 ; \mathrm{p}=0.13)$ were normally distributed by the Shapiro-Wilk test. Women comprised $23.6 \% \quad(n=34)$ of respondents, an amount significantly less than men by $t$-test $(\mathrm{p}<0.001)$. Racially, $25.0 \%(n=36)$ of the sample was nonwhite.

Cornea was the most selected subspecialization at $34.0 \%(n=49)$, while the least represented individual subspecialization was retina at $7.6 \%(n=11)$. Most surveyed ophthalmologists $(93.5 \%, \mathrm{n}=130)$ performed eye surgery, with the average respondent completing a high volume of total surgical cases in one year (1081.5 \pm 1155.1$)$. Moreover, respondents performed an average of $5424.5 \pm$ 3664.9 outpatient visits and $264.7 \pm 308.9$ in-office lasers annually. Average hours spent working amounted to $44.9 \pm$ 11.3 hours/week and 2096.6 \pm 576.1 hours/year, with 25.2 \pm 14.8 hours of the workweek spent utilizing a computer.

Table I Demographic Features of Ophthalmologists Surveyed

\begin{tabular}{|l|c|}
\hline Parameter & Value \\
\hline Age (mean years \pm SD) & $50.0 \pm 10.4$ \\
\hline Race/Ethnicity (N, \%) & \\
White & $108,75.0 \%$ \\
Asian & $26,18.1 \%$ \\
Black & $4,2.8 \%$ \\
Hispanic/Latino & $4,2.8 \%$ \\
Other & $2,1.4 \%$ \\
\hline Female (N, \%) & $34,23.6 \%$ \\
\hline Body Mass Index (mean \pm SD) & $24.4 \pm 3.4$ \\
\hline Years in Practice (mean \pm SD) & $20.8 \pm 10.4$ \\
\hline Subspecialization (N, \%) & \\
Cornea & $49,34.0 \%$ \\
Comprehensive & $43,29.9 \%$ \\
Glaucoma & $36,25.0 \%$ \\
Retina & $11,7.6 \%$ \\
Cornea and Glaucoma & $5,3.5 \%$ \\
\hline
\end{tabular}

In terms of musculoskeletal complaints, 102 respondents answered the MSK section and 72.5\% $(n=74)$ reported a history of any MSK diagnosis, while $81.4 \% \quad(n=83)$ reported MSK pain, discomfort, or disability episodes from February 2019 to February 2020. Ophthalmologists who specialized in both cornea and glaucoma reported the highest rate of prior MSK diagnoses $(100 \%, n=3)$, while cornea specialists $(66.7 \%, \mathrm{n}=26)$ and retina specialists $(66.7 \%, n=6)$ reported the lowest rates. One-year MSK complaints were most frequent in comprehensive practitioners $(88.0 \%, \mathrm{n}=22)$ and least frequent in those specializing in both cornea and glaucoma $(66.7 \%, n=2)$. Daily or weekly pain episodes were experienced by $48.1 \%(n=49)$ of the ophthalmologists surveyed. Among the 83 reporting MSK episodes, 77.1\% $(n=64)$ described their pain as chronic, with the mean duration of pain being $87.8 \pm 114.4$ months. The most common pain locations were the cervical spine $(59.0 \%, \mathrm{n}=49)$, lumbar spine $(50.6 \%, \mathrm{n}=42)$, and shoulders $(42.2 \%, \mathrm{n}=35)$ (Table 2). Least common was pain in the hips, knees, feet $(10.8 \%, n=9$ each) and elbows $(6.0 \%, n=5)$. For those reporting shoulder pain, $57.1 \%$ $(n=20)$ had a history of a shoulder diagnosis. Among thoracic or lumbar back pain sufferers, 64.8\% $(n=54)$ had a back-related diagnosis, while for cervical pain sufferers, $71.4 \%(n=35)$ reported a neck-related diagnosis. Prior diagnoses are compiled on Table 3.

Of those reporting pain, the primary sources of exacerbation were surgery $(61.4 \%, \mathrm{n}=51)$ and clinic exams $(53.0 \%, \mathrm{n}=44)$. Computer use $(32.5 \%, \mathrm{n}=27)$ and inoffice laser procedures $(28.9 \%, \mathrm{n}=24)$ were relatively less

Table 2 Body Parts Affected Among Those Reporting Pain Episodes

\begin{tabular}{|l|c|}
\hline Location of Pain $\mathbf{( N = 8 3 )}$ & $\mathbf{N}, \%^{\mathbf{a}}$ \\
\hline Cervical spine & $49,59.0 \%$ \\
\hline Lumbar spine & $42,50.6 \%$ \\
\hline Shoulders & $35,42.2 \%$ \\
\hline Thoracic spine & $22,26.5 \%$ \\
\hline Wrists and fingers & $15,18.1 \%$ \\
\hline Hips & $9,10.8 \%$ \\
\hline Knees & $9,10.8 \%$ \\
\hline Feet and toes & $9,10.8 \%$ \\
\hline Elbows & $5,6.0 \%$ \\
\hline
\end{tabular}

Note: ${ }^{a}$ Respondents could select more than one option. 
Table 3 Musculoskeletal (MSK) Diagnosis History

\begin{tabular}{|l|c|}
\hline MSK Diagnosis (N=1 02) & $\mathbf{N}, \%^{\mathbf{a}}$ \\
\hline Shoulder or rotator cuff disease or injury & $35,34.3 \%$ \\
\hline Unspecified neck pain & $31,30.4 \%$ \\
\hline Lumbar spine or lumbar disc injury or disease & $29,28.4 \%$ \\
\hline No history of MSK diagnosis & $28,27.4 \%$ \\
\hline Unspecified back pain & $26,25.5 \%$ \\
\hline Cervical spine or cervical disc injury or disease & $18,17.6 \%$ \\
\hline Other musculoskeletal disease or injury & $16,15.7 \%$ \\
\hline Lateral or medial epicondylitis & $13,12.7 \%$ \\
\hline Upper extremity arthritis & $8,7.8 \%$ \\
\hline Carpal tunnel syndrome & $5,4.9 \%$ \\
\hline Lower extremity arthritis & $5,4.9 \%$ \\
\hline Thoracic spine or thoracic disc injury or disease & $4,3.9 \%$ \\
\hline De Quervain's syndrome or trigger finger & $2,2.0 \%$ \\
\hline Bicep tendonitis & $2,2.0 \%$ \\
\hline
\end{tabular}

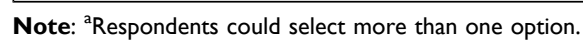

irritating. A fair portion of those with pain reported that their episodes made clinic examination $(20.5 \%, \mathrm{n}=17)$ and surgery $(21.3 \%, \mathrm{n}=17)$ moderately more difficult or worse (Figure 1). Higher TDI score, more bodily pain locations, and more MSK diagnoses were each significantly associated with increased clinic exam and surgery difficulty
(Table 4). Higher TDI score and more bodily pain locations were also significantly associated with increased inoffice laser difficulty. A few respondents $(9.6 \%, n=8)$ reported reducing the number of hours worked per week, with a median of 4.5 hours decreased, and $1.2 \%(n=1)$ of respondents stopped operating entirely due to pain. Symptoms persisted to the home environment as well, with $63.9 \%(n=53)$ of pain sufferers experiencing episodes outside of work.

The mean Total Disability Index score, inclusive of zeroes, was $6.2 \pm 7.3 \%$ out of $100 \%$. The median was $4.3 \%$. Both values fall within the minimal disability range. The highest score was $35.7 \%$, indicating moderate disability. Moderate disability scores were observed in $5.0 \%$ $(\mathrm{n}=5)$ of respondents. Comprehensive practitioners reported the highest mean TDI score at $8.9 \pm 9.1 \%$, while those who specialized in both cornea and glaucoma reported the lowest mean score at $2.4 \pm 4.1 \%$, though differences by subspecialty were non-significant $(p=0.27)$. In terms of demographics, age $(p=0.46)$, race $(p=0.65)$, height $(p=0.15)$, and years in practice $(p=0.71)$ were not significantly associated with TDI score. Annual outpatient volume $(\mathrm{p}=0.68)$, hours worked $(\mathrm{p}=1.00)$, surgical volume $(\mathrm{p}=0.94)$, and laser volume $(\mathrm{p}=0.43)$ also failed to meet significance. Sex was the closest variable to achieving significance $(\mathrm{p}=0.051)$, with a possible trend of women experiencing worse disability than men $(8.8 \pm$ $9.8 \%$ vs $5.4 \pm 6.1 \%$, respectively). Detailed findings

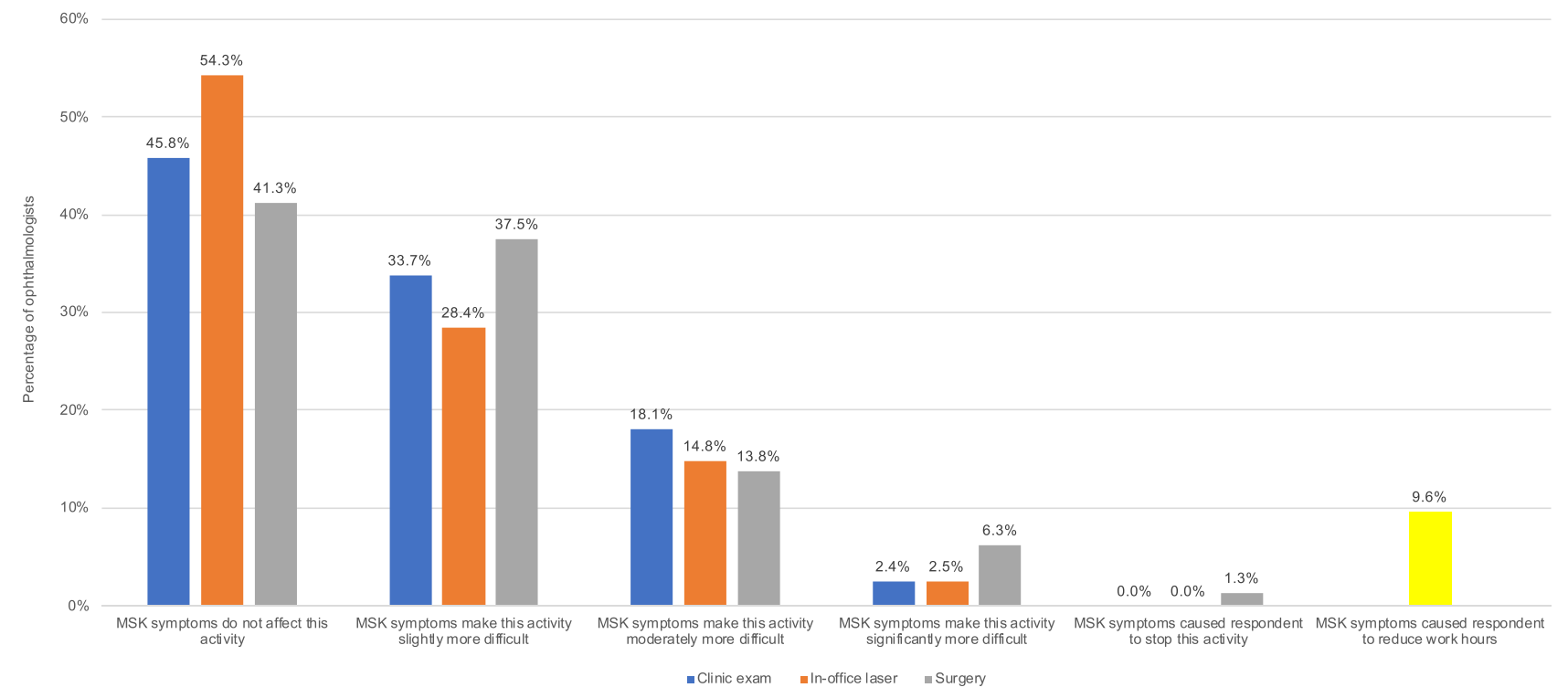

Figure I Percentage of ophthalmologists reporting clinic, laser, surgery, and work hour challenges due to musculoskeletal symptoms. Abbreviation: MSK, musculoskeletal. 
Table 4 Ordinal Regressions Testing Associations Between Musculoskeletal Complaint Characteristics and Difficulties with Ophthalmologist Job Tasks

\begin{tabular}{|c|c|c|c|c|}
\hline \multirow[t]{3}{*}{ Independent Variable } & \multicolumn{4}{|c|}{ Outcome Variable $^{a}$} \\
\hline & \multicolumn{4}{|c|}{ Clinic Exam Difficulties Attributed to Musculoskeletal Symptoms } \\
\hline & Odds Ratio & 95\% Confidence Interval & p-value & Pseudo- $\mathbf{R}^{2}$ \\
\hline Total Disability Index score & 1.131 & $0.061,0.185$ & $<0.001$ & 0.192 \\
\hline Number of pain locations & 1.697 & $1.220,2.360$ & 0.002 & 0.129 \\
\hline \multirow[t]{3}{*}{ Number of previous MSK diagnoses } & 1.363 & $1.090,1.704$ & 0.007 & 0.092 \\
\hline & \multicolumn{4}{|c|}{ In-Office Laser Difficulties Attributed to Musculoskeletal Symptoms } \\
\hline & Odds Ratio & 95\% Confidence Interval & p-value & Pseudo- $\mathbf{R}^{2}$ \\
\hline Total Disability Index score & 1.096 & $1.028,1.168$ & 0.005 & 0.105 \\
\hline Number of pain locations & 1.709 & $1.220,2.395$ & 0.002 & 0.127 \\
\hline \multirow[t]{3}{*}{ Number of previous MSK diagnoses } & 1.206 & $0.967,1.503$ & 0.097 & 0.036 \\
\hline & \multicolumn{4}{|c|}{ Surgery Difficulties Attributed to Musculoskeletal Symptoms } \\
\hline & Odds Ratio & 95\% Confidence Interval & p-value & Pseudo- $\mathbf{R}^{2}$ \\
\hline Total Disability Index score & 1.149 & I.077, 1.227 & $<0.001$ & 0.223 \\
\hline Number of pain locations & 1.808 & $1.284,2.544$ & 0.001 & 0.137 \\
\hline Number of previous MSK diagnoses & 1.284 & $1.021,1.615$ & 0.033 & 0.050 \\
\hline
\end{tabular}

Notes: a'Difficulties with clinic exam, in-office lasers, and surgery due to musculoskeletal symptoms were each self-rated with levels including "does not affect," "makes it slightly more difficult," "moderately more difficult," "significantly more difficult," and "caused me to stop this activity." Odds ratios represent the odds of reporting a more severe response on the scale, and in turn greater difficulty with the task, with a one-unit increase in the independent variable.

from the TDI-related linear regressions are included in Table 5.

The average respondent committed a total of $7.0 \pm 7.6$ hours per week to wellness activities. Specifically, $3.6 \pm$ 6.1 hours per week were spent on cardiovascular exercise, $2.0 \pm 2.3$ hours on strength training, $0.8 \pm 1.3$ hours on yoga and flexibility, and $0.6 \pm 1.4$ hours on meditation/ mindfulness. Total hours spent on wellness did not significantly correlate with TDI score $(p=0.45)$. Average job stress was rated at $4.7 \pm 1.3$ out of 7 . Based on ordinal regression, TDI score was positively $(\mathrm{OR}=1.088,95 \%$ $\mathrm{CI}=1.033,1.147)$ and significantly $(\mathrm{p}=0.001)$ associated with job stress rating. The presence of MSK pain episodes was not significantly associated with stress level $(4.8 \pm 1.2$ for those with MSK pain vs $4.2 \pm 1.6$ for those without; $p=0.054$ ), but increased frequency of pain episodes was $(\mathrm{p}=0.02)$. Those with daily pain had an average job stress score of $5.4 \pm 1.3$.

Practice modifications made and treatments used to reduce or prevent pain are compiled in Figures 2 and 3. Changing posture was the most frequent practice adjustment, with $67.7 \%(\mathrm{n}=67)$ of respondents doing so in the operating room and $64.6 \%(\mathrm{n}=64)$ in the clinic setting. Adding or changing chairs or equipment were least commonly selected. More frequent stretching $(58.6 \%, \mathrm{n}=58)$, exercise $(53.5 \%, \mathrm{n}=53)$, and over-thecounter oral medications $(50.5 \%, \mathrm{n}=50)$ were the most common treatments employed. Prescription opioids and medical marijuana were each used by only $1.0 \%(n=1)$ of respondents. The presence of MSK pain episodes was not significantly associated with making workplace adjustments $(90.0 \%, \mathrm{n}=72$ of those with pain vs $89.5 \%, \mathrm{n}=17$ of those without; $p=0.95$ ), but was significantly associated with attempting treatment $(90.0 \%, \mathrm{n}=72$ of those with pain vs $57.9 \%, \mathrm{n}=11$ of those without; $\mathrm{p}<0.001$ ). The frequency of pain episodes was significantly associated with both workplace adjustments $(\mathrm{p}=0.03)$ and attempting treatment $(\mathrm{p}=0.03) .95 .0 \%(\mathrm{n}=19)$ of those with daily pain made adjustments and $90.0 \%(\mathrm{n}=18)$ attempted treatment. In contrast, for those with pain attacks less frequent than once every few months, $50.0 \%(n=3)$ and $83.3 \%(n=5)$ made adjustments and attempted treatment, respectively. 
Table 5 Linear Regressions Testing Associations Between Ophthalmologist Characteristics and Total Disability Index Score

\begin{tabular}{|l|c|c|c|c|}
\hline Independent Variable & $\boldsymbol{\beta}$ & 95\% Confidence Interval & p-value & R-Squared \\
\hline Age & 0.054 & $-0.090,0.198$ & 0.46 & 0.006 \\
Height & 0.221 & $-0.082,0.545$ & 0.15 & 0.021 \\
Years in practice & 0.028 & $-0.119,0.174$ & 0.71 & 0.001 \\
Outpatient volume in I year & -0.00008 & $-0.0005,0.003$ & 0.68 & 0.002 \\
Hours worked in I year & -0.000006 & $-0.003,0.003$ & 1.00 & 0.000002 \\
Surgical volume in I year & 0.00005 & $-0.001,0.001$ & 0.94 & 0.00006 \\
Laser volume in I year & 0.002 & $-0.003,0.006$ & 0.43 & 0.006 \\
Weekly wellness hours & 0.072 & $-0.118,0.262$ & 0.45 & 0.006 \\
\hline
\end{tabular}

Ophthalmologists who made at least one practice modification had significantly higher TDI scores than those who $\operatorname{did}$ not $(6.7 \pm 7.5 \%$ vs $1.6 \pm 2.6 \% ; \mathrm{p}=0.03)$. Similarly, trying at least one treatment was significantly associated with a higher TDI score $(7.0 \pm 7.6 \%$ vs $2.1 \pm 3.3 \%$; $\mathrm{p}=0.01$ ).

Many respondents $(94.2 \%, \mathrm{n}=97)$ were interested in learning more about ergonomics. Some respondents (27.2\%, $\mathrm{n}=28)$ had sought the help of an ergonomics specialist, physical therapist, or occupational therapist. The most used ergonomic learning modalities were articles $(54.4 \%, \mathrm{n}=56)$ and colleagues $(53.4 \%, \mathrm{n}=55)$, while the least used were classes $(2.9 \%, \mathrm{n}=3)$ and audio $(8.7 \%, \mathrm{n}=9)$. Of those who had read an article on ergonomics, $98.1 \%$ $(\mathrm{n}=52)$ made workplace adjustments compared to $80.4 \%$ $(n=37)$ of those who did not $(\mathrm{p}=0.004)$. Other ergonomic learning modalities, including video $(\mathrm{p}=0.27)$, audio $(\mathrm{p}=0.81)$, colleagues $(\mathrm{p}=0.13)$, classes $(\mathrm{p}=0.63)$, and conferences $(\mathrm{p}=0.97)$, were not significantly associated with workplace adjustments. Having learned about ergonomics in the past was not significantly associated with interest in learning more about ergonomics $(p=0.77)$ or seeking help from an ergonomics specialist, physical therapist, or occupational therapist $(\mathrm{p}=0.60)$.

\section{Discussion}

Our findings demonstrate that MSK complaints are highly prevalent among the surveyed US ophthalmologists, with neck, low back, and shoulder pain most common. We observed that $81.4 \%$ of the respondents disclosed MSK issues, which is similar to previous survey studies that have reported MSK complaints ranging from $50.0 \%$ to $79.6 \%{ }^{1-10}$ Our percentage may lie on the higher end of reported studies because our survey asked about MSK complaints in a broad fashion ("pain, discomfort, or disability") within a 1-year time frame, and/or because our

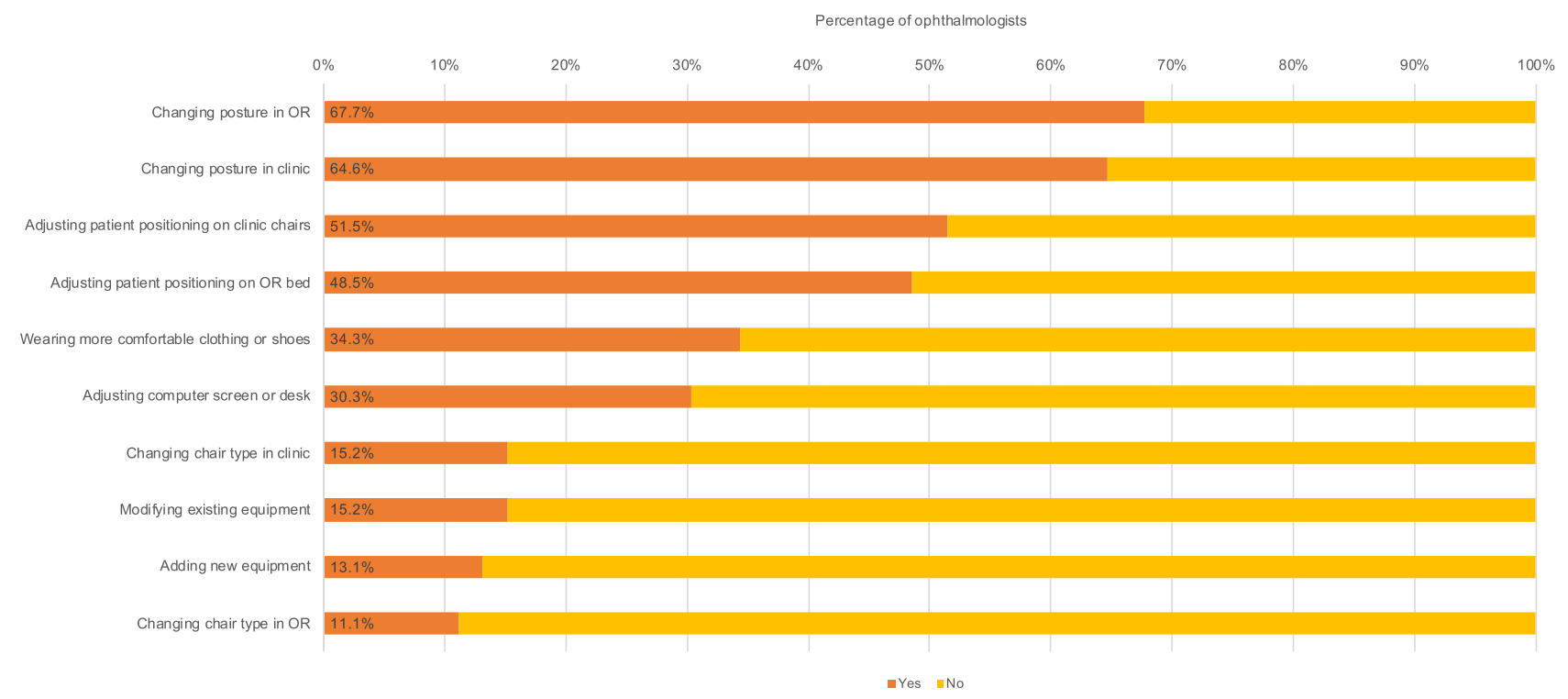

Figure 2 Percentage of ophthalmologists who made various workplace adjustments to prevent or reduce musculoskeletal symptoms. Abbreviation: OR, operating room. 


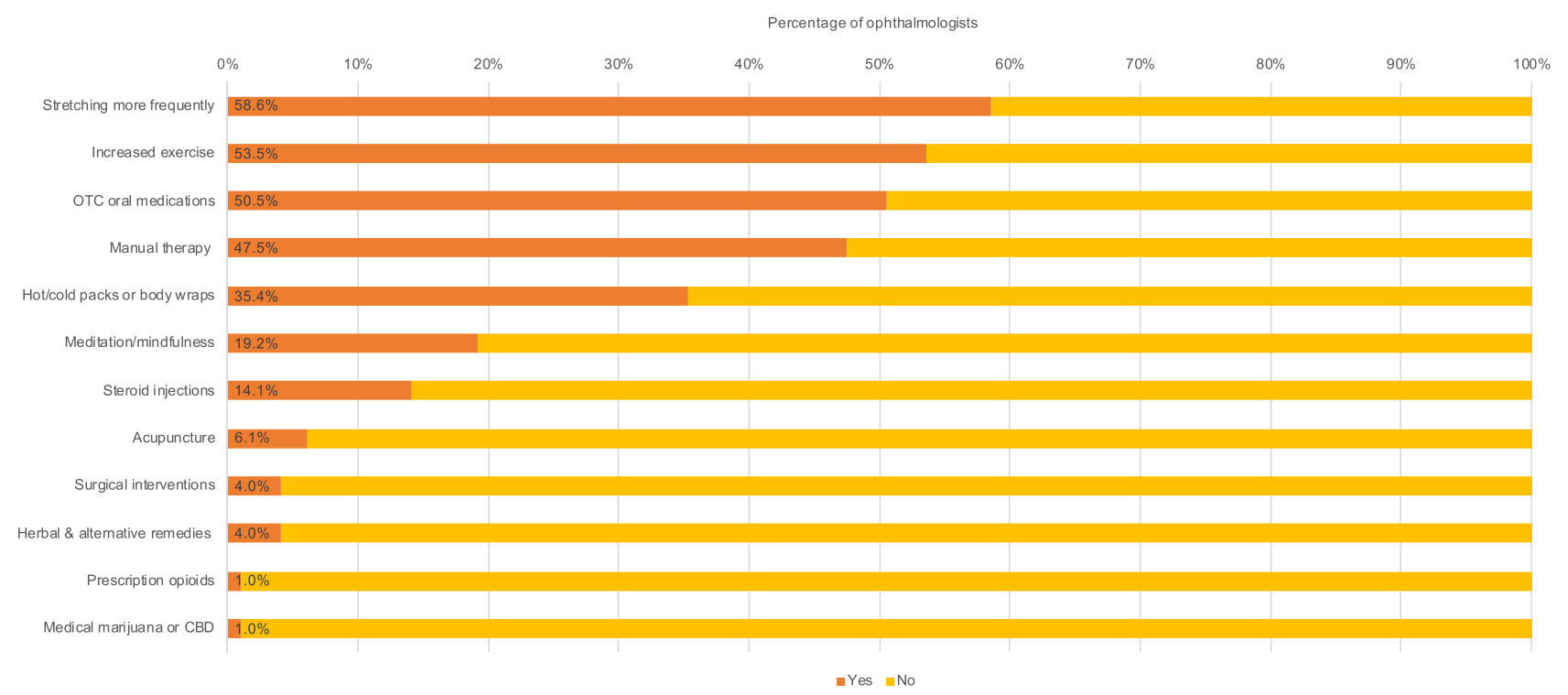

Figure 3 Percentage of ophthalmologists who used various treatments to prevent or reduce musculoskeletal symptoms. Abbreviations: CBD, Cannabidiol; OTC, Over-the-counter.

sample included many high volume practitioners in terms of surgery and clinic visits.

Our study is the first in the ophthalmic literature to use a statistically validated scale that quantifies the extent of disability associated with the entire spine. The average respondent in our sample had only minimal disability relating to the neck or back based on TDI score, with symptoms being commonly exacerbated by surgery and clinic examinations. Previous studies concur with these results, generally finding that most ophthalmologist pain sufferers report mild severity despite ample workplace irritations. ${ }^{1,2,4,5}$ However, there were a few respondents in our sample who earned moderate disability scores. Further, pain made performing clinic examinations or surgeries moderately more difficult or worse for around $20 \%$ of those reporting symptoms, with $9.6 \%$ of sufferers reducing their work hours and $1.2 \%$ eliminating surgery from their practice. These values are comparable to the findings of Schechet et al 2021 in a sample containing multiple ophthalmology subspecialties. ${ }^{9}$ A separate study of oculoplastic surgeons reported that $9.2 \%$ of its sample had ended their surgical careers due to pain, a notably higher amount. ${ }^{10}$ It may be the case that loupe and headlamp use may cause greater musculoskeletal impairment than surgical microscopy.

Our sample conducted a high number of average annual clinic visits (5424.5) and surgical cases (1081.5), yet neither volume correlated with the extent of disability relating to the neck and back. This may be due to our survey only taking a 1-year snapshot of practice volumes. Nonetheless, this lack of significant association follows a pattern seen in multiple previous surveys. Chatterjee et al 1994, Dhimitri et al 2005, Diaconita et al 2018, and Hyer et al 2015 each found that volume metrics such as number of cataract surgeries or hours spent in clinic were not predictive of MSK symptoms. ${ }^{3-6}$ In contrast, Kitzmann et al 2012 found that number of patients seen per day was significantly associated with neck symptoms, and Schechet et al 2021 found that time spent in surgery was positively correlated with pain severity. ${ }^{8,9}$ Ambivalent findings may be attributable to a variety of factors. It may be the case that some ophthalmologists feel increased pain while still maintaining a greater workload, while other ophthalmologists have already decreased their practice and surgery volumes prior to being surveyed due to pain, causing a net null calculation. Alternatively, volume metrics may be less impactful than ophthalmologists' ergonomic habits. Kaup et al 2020 found that good ergonomic practices in the clinic and OR, such as stretching, maintaining spine neutrality, and ensuring appropriate chair heights, were associated with fewer MSK symptoms. ${ }^{7}$ Perhaps the ideal method to establish the effect of volume metrics on MSK symptoms would be to account for lifetime clinic and surgical numbers, and to define the extent of disability longitudinally. However, this approach would be challenging given recall bias and lack of documentation. 
Nearly half of the sample (48.1\%) reported daily or weekly pain episodes, a rate that is within the range of prior reports. ${ }^{6,9}$ Despite the high frequency of symptoms, our study found that there may be an underdiagnosis of MSK diseases in ophthalmologists, especially for shoulder pathologies. Slightly over half (54.2\%) of those reporting shoulder pain had a corresponding diagnosis, versus $64.8 \%$ with back pain and $71.4 \%$ with neck pain. This may be explained by mostly mild impairment in functioning. Ophthalmologists without more severe or disabling symptoms may not actively seek diagnostic evaluation. For comparison, Kitzmann et al 2012 found that $23 \%$ of ophthalmologists had an official back pain diagnosis compared to $24 \%$ who reported low back pain, $13 \%$ slipped/ ruptured cervical disc diagnosis vs $46 \%$ self-reported neck pain, and $12 \%$ rotator cuff tendonitis diagnosis vs $11 \%$ self-reported shoulder pain. ${ }^{8}$ What may explain the difference compared to our results is that prior studies did not specifically examine whether the individuals reporting current pain at those locations were also the same subjects reporting a history of corresponding diagnoses.

Job-related stress scores were positively associated with TDI scores and pain frequency in our sample. Furthermore, those with higher TDI scores, more body parts with pain, and more past MSK diagnoses were more likely to report difficulties while operating and examining patients in clinic. Given that those with greater physical disability experienced greater mental strain and job task challenges, it may be the case that MSK symptoms play a role in ophthalmologist burnout. ${ }^{15}$ Our findings are supported by those of Dhimitri et al 2005, who found that American ophthalmologists reporting pain symptoms in the neck and back had higher stress scores. ${ }^{4}$

Most of our sample had made at least one practice adjustment or attempted one form of treatment to prevent or reduce MSK symptoms. As observed in prior surveys, over-the-counter medications were a popular option, while surgeries and opioid use was seldom required. ${ }^{1,3,5,6,9}$ The high frequency of postural changes and stretching aligns with prior claims that "working in the same position for long periods" and "working in awkward or cramped positions" contribute to ophthalmologists' pain. ${ }^{8}$ Furthermore, respondents in our study with greater spine-related disability were more likely to pursue practice modifications or attempt treatments. Combined with our finding that $94.2 \%$ of respondents are interested in learning more about ergonomics, it appears that there is considerable demand in ophthalmology for pain relief and MSK prophylaxis strategies.

Our study had a reasonably good response rate compared to other web-based surveys of specialists, which typically average $35.0 \% .{ }^{16} \mathrm{~A}$ disadvantage of our study is the limited sample size, with relatively fewer female and racial minority respondents. The lack of female representation may have led to insufficient power when comparing TDI scores; one study with a larger sample size found that female ophthalmologists were more likely to experience neck and upper extremity symptoms. ${ }^{4}$ However, other similar surveys in ophthalmology have reported less than a third of female respondents. ${ }^{6,10}$ As with any survey study, ours is subject to recall bias, especially with regard to questions that asked about practice or surgical volumes from months prior. ${ }^{17}$ It is possible that recall bias contributed to the high standard deviations for laser and surgical cases. Furthermore, the cross-sectional design makes it difficult to establish causal relationships. Our study excluded fields in ophthalmology such as neuroophthalmology and oculoplastics to streamline question types. Inclusion of these subspecialties may have elucidated additional insight. Furthermore, in terms of sampling technique, the reliance on personal networks and nearby professional organizations for email lists may have led to US Northeast geographic biases.

Future research should explore practical approaches to alleviating and preventing musculoskeletal complaints in the ophthalmologist population. Prospective longitudinal studies could be performed that compare specialist support, such as occupational therapy counseling versus no counseling, to determine any advantages in symptom frequency, symptom severity, and job task impact over a year or more. Similar comparisons could be performed to evaluate specific bodily interventions such as a stretching routine or exercise routine against no regimen. Given that respondents in the current study were more likely to pursue practice modifications if they had read articles on ergonomics, it may be valuable to prospectively examine which methods of ergonomic instruction are most effective at generating beneficial practice changes. Another aspect to analyze would be the impact of novel technological approaches. For example, two recent survey studies have elucidated potential ergonomic benefits to the use of heads-up displays in the ophthalmologist operating suite. ${ }^{18,19}$ Additionally, to better appreciate the scope of the MSK problem in ophthalmology compared to the general population, future surveys could enlist randomly 
selected control groups composed of non-ophthalmologists that would be asked identical symptom-related questions.

\section{Conclusion}

MSK complaints continue to be prevalent among surveyed US ophthalmologists. The sample better reflected practitioners in the Northeast, but a similar trend could be expected for other US ophthalmologists. Though extent of functional impairment tends to be minimal, many practitioners report frequent pain, discomfort, or disability episodes. More severe disability and higher frequency of pain attacks correlate with greater job stress, which may be implicated in physician burnout. Furthermore, there may be both underdiagnosis of some pathologies and considerable demand for options to treat and prevent MSK symptoms. Overall, there is a need for further, prospective research to explore practical methods of addressing MSK complaints among ophthalmologists.

\section{Acknowledgments}

This paper was presented at the podium of the American Society of Cataract and Refractive Surgeons (ASCRS) 2021 Annual Meeting with interim findings. The presentation's abstract was published in the "Abstracts" section of the ASCRS Clinical Education webpage (https://ascrs.org/ clinical-education/abstracts). This study was funded through an investigator-initiated trial grant (IIT \#61071789) from the Alcon Research Institute (Fort Worth, TX, USA). The authors would like to thank the Alcon Research Institute for the grant. The authors would also like to thank all of the ophthalmologists who generously gave their time to complete the survey questions.

\section{Disclosure}

EDR is a consultant to Alcon and Glaukos. PKG is a consultant to: Alcon, Allergan, HanAll Biopharma, J\&J Vision, Kala, New World Medical, Novartis, Ocular Therapeutix, Oyster Point, ReGenTree, Sight Sciences, Sun Pharmaceuticals, Tear Lab, Tissue Tech Inc., and Zeiss. NMR reports the following: CATS LLCConsultant (C). Allergan-C, Speaker (S). Alcon-C, S. Novartis - C, S. Ellex-C. Eyenovia-C. LumenisC. ELT Sight $-C$, Equity (E). Equinox-C, Investor (I). Iridex-C. Aerie - C, S. Bausch \& Lomb/Valeant-C, S. BVI-C, S. Eyepoint-C. Ivantis - C, S. Glaukos-C, S. Alimera-C, S. Kala Lumenis Inc-C, S, New World Medical-C, S. SpyGlass-C. Sight Sciences-C, S. Santen, Inc - C. Thea - C. Reichert - C. Shire-C.
Carl Zeiss Meditec-C. Ocular Science-C. Ocular Therapeutix-C. Omeros-C. Quantel Medical-C. ViaLase - C. The authors report no other conflicts of interest in this work.

\section{References}

1. Al-Marwani Al-Juhani M, Khandekar R, Al-Harby M, et al. Neck and upper back pain among eye care professionals. Occup Med. 2015;65(9):753-757.

2. Chams H, Mohammadi SF, Moayyeri A. Frequency and assortment of self-report occupational complaints among Iranian ophthalmologists: a preliminary survey. MedGenMed. 2004;6(4):1.

3. Chatterjee A, Ryan WG, Rosen ES. Back pain in ophthalmologists. Eye. 1994;8(Pt 4):473-474. doi:10.1038/eye.1994.112

4. Dhimitri KC, McGwin G Jr, McNeal SF, et al. Symptoms of musculoskeletal disorders in ophthalmologists. Am J Ophthalmol. 2005;139 (1):179-181. doi:10.1016/j.ajo.2004.06.091

5. Diaconita V, Uhlman K, Mao A, et al. Survey of occupational musculoskeletal pain and injury in Canadian ophthalmology. Can J Ophthalmol. 2019;54(3):314-322. doi:10.1016/j.jcjo.2018.06.021

6. Hyer JN, Lee RM, Chowdhury HR, et al. National survey of back \& neck pain amongst consultant ophthalmologists in the United Kingdom. Int Ophthalmol. 2015;35(6):769-775. doi:10.1007/ s10792-015-0036-z

7. Kaup S, Shivalli S, Kulkarni U, et al. Ergonomic practices and musculoskeletal disorders among ophthalmologists in India: an online appraisal. Eur J Ophthalmol. 2020;30(1):196-200. doi:10.1177/1120672118815107

8. Kitzmann AS, Fethke NB, Baratz $\mathrm{KH}$, et al. A survey study of musculoskeletal disorders among eye care physicians compared with family medicine physicians. Ophthalmology. 2012;119 (2):213-220. doi:10.1016/j.ophtha.2011.06.034

9. Schechet SA, DeVience E, DeVience S, et al. Survey of musculoskeletal disorders among US ophthalmologists. Digit J Ophthalmol. 2021;26(4):36-45. doi:10.5693/djo.01.2020.02.001

10. Sivak-Callcott JA, Diaz SR, Ducatman AM, et al. A survey study of occupational pain and injury in ophthalmic plastic surgeons. Ophthalmic Plast Reconstr Surg. 2011;27(1):28-32. doi:10.1097/ IOP.0b013e3181e99cc8

11. Lakhiani C, Fisher SM, Janhofer DE, et al. Ergonomics in microsurgery. J Surg Oncol. 2018;118(5):840-844. doi:10.1002/ jso. 25197

12. Howarth AL, Hallbeck S, Mahabir RC, et al. Work-related musculoskeletal discomfort and injury in microsurgeons. J Reconstr Microsurg. 2019;35(5):322-328. doi:10.1055/s-0038-1675177

13. Spiegel MA, Lafage R, Lafage V, et al. Developing the total disability index based on an analysis of the interrelationships and limitations of Oswestry and neck disability index. Spine. 2016;41(1):74-81. doi:10.1097/BRS.0000000000001159

14. Cruz DL, Ayres EW, Spiegel MA, et al. Validation of the recently developed total disability index: a single measure of disability in neck and back pain patients. J Neurosurg Spine. 2019:1-9. doi:10.3171/ 2019.9.SPINE19331

15. Viviers S, Lachance L, Maranda MF, et al. Burnout, psychological distress, and overwork: the case of Quebec's ophthalmologists. Can J Ophthalmol. 2008;43(5):535-546. doi:10.3129/i08-132

16. Cunningham CT, Quan H, Hemmelgarn B, et al. Exploring physician specialist response rates to web-based surveys. BMC Med Res Methodol. 2015;15:32. doi:10.1186/s12874-015-0016-z

17. Althubaiti A. Information bias in health research: definition, pitfalls, and adjustment methods. J Multidiscip Healthc. 2016;9:211-217. doi:10.2147/JMDH.S104807 
18. Bin Helayel H, Al-Mazidi S, AlAkeely A. Can the three-dimensional heads-up display improve ergonomics, surgical performance, and ophthalmology training compared to conventional microscopy? Clin Ophthalmol. 2021;15:679-686. doi:10.2147/OPTH.S290396
19. Weinstock RJ, Ainslie-Garcia MH, Ferko Nc, et al. Comparative assessment of ergonomic experience with heads-up display and conventional surgical microscope in the operating room. Clin Ophthalmol. 2021;15:347-356. doi:10.2147/OPTH.S292152

\section{Publish your work in this journal}

Clinical Ophthalmology is an international, peer-reviewed journal covering all subspecialties within ophthalmology. Key topics include: Optometry; Visual science; Pharmacology and drug therapy in eye diseases; Basic Sciences; Primary and Secondary eye care; Patient Safety and Quality of Care Improvements. This journal is indexed on PubMed

Submit your manuscript here: https://www.dovepress.com/clinical-ophthalmology-journal
Central and CAS, and is the official journal of The Society of Clinical Ophthalmology (SCO). The manuscript management system is completely online and includes a very quick and fair peer-review system, which is all easy to use. Visit http://www.dovepress.com/ testimonials.php to read real quotes from published authors. 